\title{
SOME CONDITIONS FOR FINITENESS AND COMMUTATIVITY OF RINGS
}

\author{
HOWARD E. BELL and FRANCO GUERRIERO \\ Department of Mathematics \\ Brock University \\ St. Catharines, antario, Canada L2S 3Al \\ (Received March 21, 1989 and in revised form September 9, 1989)
}

ABSTRACT. We present several new sufficient conditions for a ring to be finite; we give two conditions which for periodic rings $\mathrm{R}$ imply that $\mathrm{R}$ must be either finite or commutative; and we study commutativity in rings with only finitely many non-central subrings.

KEY WORDS AND PHRASES. Finiteness of rings, perlodic rings, commutativity. 1980 AMS SUBJECT CLASSIFICATION CODES. 16A44, 16A7O.

\section{INIRODUCTION.}

Over the years, several authors have given sufficient conditions for a ring to be finite, these conditions typically involving restrictions on subrings or zero divisors. More recently, Putcha and Yaqub [1] provided a sufficient condition for finiteness of a non-nil ring - specifically that the set of non-nilpotent elements be finite; and Bell [2] presented conditions implying that a ring is either cormutative or finite.

In this paper, we offer some new conditions for a ring to be finite, and continue the development of the cormutative-or-finite theme. Some of our results are extensions of known results; others, particularly those in the final section, are of a quite different character.

In what follows, $R$ is an associative ring with center $C$. The set of nilpotent elements is denoted by $\mathrm{N}$; and for a subset $\mathrm{S}$ of $\mathrm{R}$, the subring generated by $\mathrm{S}$ is denoted by $\langle\mathrm{S}\rangle$. The term zero divisor will mean a one-sided zero divisor (i.e. not necessarily a two-sided zero divisor), and 0 will be considered a zero divisor. For $x \in R$, the symbols $A_{r}(x), A_{l}(x)$, and $A(x)$ denote respectively the right, left, and two-sided annihilators of $x$. Finally, the symbols $z, z_{n}$, and $C\left(p^{\infty}\right)$ denote respectively the ring of integers, the ring of integers mod. $n$, and the Prüfer p-group.

We shall frequently have use for direct-sum decompositions of $R$, both as a ring and as an additive group. To make the distinction clear, we use the symbol $\oplus$ to denote a ring-theoretic direct sum and the symbol $\tilde{+}$ to denote an additive-group direct sum.

2. A FTNITENESS RESULT.

We begin by discussing rings in which certain subrings of zero divisors are assumed to be finite. 
THEOREM 1. Let $\mathrm{R}$ be a ring with at least one non-nilpotent zero divisor, and suppose that every proper non-nil subring of zero divisors is finite. Then either (i) $R$ is finite, or (ii) there exist primes $p$ and $q$, not necessarily distinct, and a positive integer $k$, such that $R=z_{q} k T$, where $\mathrm{T}$ is the zero ring on $\mathrm{C}\left(\mathrm{p}^{\infty}\right)$.

PROOF. Let $x$ be a non-nilpotent zero divisor. If $\left\langle x^{2}\right\rangle \neq R$, then $\left\langle x^{2}\right\rangle$ is finite and there exist distinct positive integers $n$ and $m$, with $n>m$, such that $x^{n}=x^{m}$. A standard computation (see [3]) shows that $e=x^{m(n-m)}$ is a non-zero idempotent zero divisor. On the other hand, if $\left\langle x^{2}\right\rangle=R$, then $\mathrm{x}=\mathrm{x}^{2} \mathrm{p}(\mathrm{x})$, where $\mathrm{p}(\mathrm{X}) \in \mathrm{z}[\mathrm{X}]$; and $\mathrm{xp}(\mathrm{x})$ is a non-zero idempotent zero divisor. In any case, $\mathrm{R}$ contains a non-zero idempotent zero divisor $\mathrm{e}$, which without loss we assume to be a left zero divisor.

Both of the subrings $e R$ and $A_{r}(e)$ are proper and we have the grouptheoretic direct sum

$$
R=e R \tilde{+} A_{r}(e) .
$$

If $A_{\ell}(e) \neq\{0\}$, then $e R$ is a proper non-nil subring of zero divisors. If $A_{\ell}(e)=\{0\}$, then $e R=e R e$ and again $e R$ is a proper non-nil subring of zero divisors. Therefore $\mathrm{eR}$ is always finite. Moreover, if $\mathrm{A}_{r}(e)$ is non-nil, then it is finite and so is R. Thus, assume that $A_{r}(e)$ is nil; and consider $\left\langle e, A_{r}(e)\right\rangle$, which is clearly a non-nil subring of $R$. If $y \in\left\langle e, A_{r}(e)\right\rangle$, then $y=n e+x$ where $n$ is an integer and $x \in A_{r}(e)$; and choosing $m>1$ such that $x^{m}=0 \neq x^{m-1}$, we see that $y x^{m-1}=0$, so that $\left\langle e, A_{r}(e)\right\rangle$ is a non-nil subring of zero divisors. If it is a proper subring, it is finite, in which case $A_{r}(e)$ is finite and $R$ is finite.

Therefore we can suppose that $\left\langle e, A_{r}(e)\right\rangle=R$, and that $A_{r}(e)$ is $n i l$ and infinite. We now have the group-theoretic direct sum

$$
A_{r}(e)=A_{r}(e) e \tilde{+} A(e) \text {. }
$$

we claim that $A_{r}(e) e$ is finite. Consider $S=\left\langle e, A_{r}(e) e\right\rangle$, which is a non-nil subring of zero divisors. Since $\langle e\rangle$ is a subring of the finite ring eR, e must have finite additive order. Moreover, e is a right identity element for $S$, and hence $(S,+)$ is a periodic abelian group of bounded order; therefore, every subgroup of $(S,+)$ is a direct sum of cyclic groups [4, p. 44]. Let $B=\left\{x_{\alpha} \mid \alpha \in \Lambda\right\}$ be a basis for the group $\left(A_{r}(e) e,+\right)$, and hence $\{e\} \cup \mathrm{B}$ a basis for $\mathrm{S}$. Choose $\mathrm{x}_{\alpha_{0}} \in \mathrm{B}$, and define $\mathrm{B}_{1}$ to be $\mathrm{B} \backslash \mathrm{x}_{\alpha_{0}}$. Then $S_{1}=\left\langle e, B_{1}\right\rangle$ is a proper non-nil subring of zero divisors; therefore $B_{1}$ is finite, $B$ is finite, and $A_{r}(e) e$ is finite. Since $A_{r}(e)$ was infinite and nil, we know by (2.1) that $A(e)$ is nil and infinite; consequently, $A(e)$ 
contains an infinite zero ring $T$ [5, Proposition 5].

Recall that $R=e R \tilde{+} A_{r}(e) e \tilde{+} A(e)$. If $A_{r}(e) e \neq\{0\}$, then $\langle e\rangle \tilde{+} T$ is $a$ proper infinite non-nil subring of zero divisors, contrary to our

hypothesis. Thus $R=e R \tilde{+} A(e)$. Moreover $e R=\langle e\rangle$ and $A(e)=T$, for otherwise $\langle e\rangle \tilde{+} T$ violates our hypothesis again. It is now immediate that $R$ is a ring-theoretic direct sum of $\langle e\rangle$ and $A(e)$.

our basic finiteness hypothesis on $R$ forces every proper subring of $A(e)$ to be finite, and a result of Laffey [6] implies that $A(e)$ must be the zero ring on $C\left(p^{\infty}\right)$ for some prime $p$. It is clear that $\langle e\rangle=z_{n}$ for some positive integer $n$. If $n=j k$, where $j$ and $k$ are relatively prime and greater than 1 , then $z_{n}=z_{j} \oplus z_{k}$, and $z_{k} \oplus T$ violates our hypothesis on $R$. Therefore $\langle e\rangle=z_{q} k$ for some prime $q$ and positive integer $k$.

An immediate consequence of Theorem 1 is the following:

COROLLARY 1. Let $R$ have at least one non-nilpotent zero divisor, and assume that every non-nil subring of zero divisors is finite. Then $R$ is finite.

3. FINITE OR COMMTATIVE PERTODIC RINGS.

We now turn our attention to periodic rings, and prove two results on the theme of commutativity and finiteness. The first is motivated by Bell's result that a periodic ring with only a finite number of non-central zero divisors must be commutative or finite [2, Theorem 3].

THEOREM 2. A periodic ring $\mathrm{R}$ with only finitely many non-central subrings of zero divisors is finite or commutative.

Before beginning the proof, we recall a useful fact about periodic rings, namely that they are either nil or possess non-zero idermotents. Indeed same power of each element is idempotent [3], so that a periodic ring having non-nilpotent zero divisors has a non-zero idempotent zero divisor.

There does not seem to be a short proof of Theorem 2, so we separate out same of the details in four initial lemas. The first is a well-known result of Herstein; the second is due to Szele.

LEMA 1 ([7], [8]). If $R$ is a periodic ring all of whose nilpotent elements are central, then $R$ is commutative.

LEMMA 2 [9]. A ring $R$ having both ascending chain condition and descending chain condition on subrings must be finite. In particular, any ring with only a finite number of subrings is finite.

LEMMA 3. Let $R$ be a ring with only finitely many non-central subrings of zero divisors. Then any non-central nilpotent element has finite additive order.

PROOF. Let $u$ be a non-central nilpotent element of $R$ with $u^{m}=0 \neq u^{m-1}$ for some positive integer $m>1$. Note that there is at most one prime $p$ for which pu is central, hence there exists a prime $P$ such that for all primes $\mathrm{p} \geq \mathrm{P}, \mathrm{pu} \notin \mathrm{C}$. There must exist distinct primes $\mathrm{p}$ and $\mathrm{q}$ such that $\mathrm{p}, \mathrm{q} \geq \mathrm{P}$ 
and $\langle$ pu $\rangle=\langle q u\rangle$, and thus there exist integers $n_{1}, n_{2}, \ldots, n_{k}$ such that

$$
\mathrm{pu}=\mathrm{n}_{1} q \mathrm{u}+\mathrm{n}_{2} \mathrm{u}^{2}+\ldots+\mathrm{n}_{\mathrm{k}} \mathrm{u}^{\mathrm{k}} \text {. }
$$

Hence, $t u=n_{2} u^{2}+\ldots+n_{k} u^{k}$, where $t=p-n_{1} q \neq 0$. consequently,

$$
t^{2} u=n_{2} u(t u)+n_{3} u^{2}(t u)+\ldots+n_{k} u^{k-1}(t u) .
$$

The right side of (3.1) is a polynomial in $u$, with each term of degree at least three. By continuing in this manner, we see that $t^{m-1} u=0$.

LEMMA 4. Let the periodic ring $R$ have only finitely many non-central subrings of zero divisors.

(i) If $e$ is an idempotent and $e R$ is not cormutative, then $A(e)$ is finite.

(ii) If $R$ is the ring-theoretic direct sum of $R_{1}$ and $R_{2}$, with $R_{1}$ non-camutative, then $R_{2}$ is finite.

(iii) If $e$ is a non-central idempotent of $R$, then $A_{r}(e) e$ is finite.

(iv) If $e$ is a non-central idempotent of $R$ such that $e R$ is commutative and $A_{r}(e) e \nsubseteq C$, then $e R$ is finite.

PROOF. (i) Since $e R$ is not commutative, there exists a nilpotent element $u$ in $e R$ which is not central in $e R$, hence not central in $R$. Let $u^{k}=0 \neq u^{k-1}$, for some positive integer $k>1$. For any subring $S$ of $A(e)$, $\langle\mathrm{u}, \mathrm{S}\rangle \mathrm{u}^{\mathrm{k}-1}=\{0\}$, hence $\langle\mathrm{u}, \mathrm{S}\rangle$ is a non-central subring of zero divisors, and therefore there are only finitely many such subrings $\langle u, S\rangle$. Suppose that $S_{1}$ and $S_{2}$ are subrings of $A(e)$ such that $\left\langle u, S_{1}\right\rangle=\left\langle u, S_{2}\right\rangle$. Then for arbitrary $s_{1}$ in $s_{1}$, we can write

$$
s_{1}=p(u)+s_{2}+\sum_{i=1}^{r} q_{i}(u) t_{i} \text { for same positive integer } r,
$$

where $s_{2}$ and the $t_{i}$ are in $s_{2}$, and $p(x)$ and the $q_{i}(x)$ are in $x z[x]$.

Thus,

$$
s_{1}-s_{2}=p(u)+\sum_{i=1}^{r} q_{i}(u) t_{i}
$$

which is in $A(e) \cap e R=\{0\}$. It follows that $S_{1} \subseteq S_{2}$, and similarly ane shows that $S_{2} \subseteq S_{1}$. Therefore, $A(e)$ has only a finite number of subrings, and hence is finite by Lemma 2 .

(ii) The argument is similar to that of (i) and is amitted.

(iii) We may assume that $A_{r}(e) e \neq\{0\}$; moreover, $A_{r}(e) e$ is a zero ring. If $S$ is any subring of $A_{r}(e) e$, then $\langle e, S\rangle A_{r}(e) e=\{0\}$, so $\langle e, S\rangle$ is a non-central subring of zero divisors. By hypothesis, there are only finitely many such subrings; and furthermore, it is even easier than in part (i) to show that different $S$ give rise to different $\langle e, S\rangle$. Thus $A_{r}(e) e$ has only finitely many subrings and must therefore be finite, again by Lerma 2. 
(iv) Choose $w \in A_{r}(e) e l c$. Since $e R$ is commutative, $e R=e R e$, so that $e R A_{r}(e)=\{0\}$; therefore, for any subring $T$ of $e R,\langle w, T\rangle w=\{0\}$ and $\langle w, T\rangle$ is non-central. As usual, this implies there are only finitely many such $\mathrm{T}$; and $\mathrm{eR}$ is finite.

PROOF OF THECREM 2. In view of Lemma 1 we may assume that $R$ contains a non-central nilpotent element $x$. Then $\langle x\rangle$ is a non-central subring of zero divisors, and it is finite by Lemma 3. Since $R$ has only finitely many non-central subrings of zero divisors, we see that if each non-central zero divisor is nilpotent, then the set of non-central zero divisors is finite and by Bell's result [2] $R$ is finite or commutative.

Henceforth we may assume that $R$ contains a non-central, non-nilpotent zero divisor. Therefore, $\mathrm{R}$ contains a non-zero idempotent zero divisor $\mathrm{e}$, which we assume without loss to be a left zero divisor.

Let $n(R)$ denote the mumber of proper non-central subrings of zero divisors. Assume now that the conclusion is false and that $R$ is $a$ counterexample with $n(R)$ minimal among counterexamples.

If $e \in C$, then $R$ is the ring-theoretic direct sum of $e R$ and $A(e)$, with one of these non-camutative. Since both $e R$ and $A(e)$ are proper subrings of zero divisors, we have that $n(e R), n(A(e))<n(R)$. Whichever of these is non-commutative must be finite; and by Lerma 4 (ii), the other is finite as well.

Therefore $e \notin C$, and we assume first that $A(e) \neq\{0\}$. Then $e R$ and $R e$ are both proper non-central subrings of zero divisors; moreover, if both were commutative, we would have ex $=$ exe $=$ xe for all $x \in R$, contrary to the fact that $e \notin C$. Thus we may assume that $e R$ is not camutative and $n(e R)<n(R)$, so the minimality of $n(R)$ implies that $e R$ is finite. By Lemma 4 (i) and (iii), both $A_{r}(e) e$ and $A(e)$ are finite, hence $R=e R \tilde{+} A_{r}(e) e \tilde{+A}(e)$ is also finite.

Finally we assume that $e \notin C$ and $A(e)=\{0\}$. Then $R=e R \tilde{+} A_{r}(e) e$, with the latter summand being finite by Lemma 4 (iii). If $A_{r}(e) e \subseteq C$, then $A_{r}(e) e=\{0\}$, contradicting our assumption that $A(e)=\{0\}$. Thus, assume that $A_{r}(e) e \notin C$, in which case $n(e R)<n(R)$ again. Therefore, $e R$ is again finite or commutative; and in view of Lerma 4 (iv), $e R$ is finite in any case, showing that no counterexample can exist.

THEOREM 3. Let $R$ be a periodic ring. If every proper non-central subring of zero divisors is finite, then $R$ is finite or camutative.

PROOF. Suppose first that all elements of $R$ are zero divisors, in which case every proper non-central subring of $R$ is finite. If $R$ is not commutative and $x \in R \backslash C$, then $\langle x, C$ is proper and non-central, hence finite; therefore, all commutative subrings of $R$ are finite, and $R$ is finite by $a$ theorem of Laffey [10]. 
Henceforth we may assume that $R$ contains regular elements. If all zero divisors are nilpotent, the fact that $\mathrm{R} \neq \mathrm{N}$ guarantees that $\mathrm{R}$ has non-zero idempotents, all of which are regular. Therefore $R$ contains a unique non-zero idermpotent, which must be 1; and it follows that every element of $\mathrm{R}$ is either nilpotent or invertible, a sufficient condition for $\mathrm{N}$ to be an ideal [11]. If $N \subseteq C$, then $R$ is camutative by Lerma 1 . Otherwise, $N$ is a proper non-central subring, hence is finite; therefore $R$ has only a finite number of zero divisors and is finite by an old theorem of Ganesan ([12], [13]).

We can now assume that $\mathrm{R}$ contains a non-nilpotent zero divisor and hence a non-zero idempotent zero divisor $e$, which we assume to be a left zero divisor.

As usual we write $R=e R \tilde{+} A_{r}(e)$. If $A_{\ell}(e) \neq\{0\}$, then $e R$ consists of zero divisors; and if $A_{\ell}(e)=\{0\}$, then $e R=e R e$ and $e R A_{r}(e)=\{0\}$. In any case $e R$ and $A_{r}(e)$ are both proper subrings of zero divisors. If both are central, then $R$ is commutative; otherwise, at least one is finite by hypothesis, and arguments similar to those used in proving Lerma 4 establish finiteness of the other as well.

4. RINGS WITH FINITELIY MANY NON-CENIRAL SUBRINGS.

Theorem 2 of this paper suggests that we should examine arbitrary rings with only a finite mumber of non-central subrings. We now present same results on such rings, and note that the first is a refinement of a theorem of Bell [14], which asserts that a ring with fewer than three proper subrings is commutative.

THEOREM 4. If $R$ has fewer than four non-central subrings, then $R$ is cormutative.

PROOF. Let $R$ be a non-cormutative ring. Then there exist $x, y \in R \backslash C$ such that $[x, y] \neq 0$. Note that $\langle x, C\rangle$ and $\langle y, C\rangle$ are cormutative and hence proper; moreover $\langle x, C\rangle\langle y, C\rangle$, for otherwise we would have $[x, y]=0$. Since $(R,+)$ cannot be the union of two proper subgroups, there exists $z \notin\langle\mathrm{x}, \mathrm{C}\rangle \cup\langle\mathrm{y}, \mathrm{C}\rangle$. Clearly $\langle z, \mathrm{C}\rangle$ is a proper subring different from both $\langle\mathrm{x}, \mathrm{C}\rangle$ and $\langle\mathrm{y}, \mathrm{C}\rangle$; hence, we have now exhibited four non-central subrings, including $\mathrm{R}$ itself.

As the following example demonstrates, Theorem 4 is best possible. Let $(R,+)=\{0, a, b, c\}$ be the Klein four-group and define multiplication by $0 \mathrm{x}=\mathrm{x} 0=\mathrm{cx}=0, \mathrm{ax}=\mathrm{bx}=\mathrm{x}$ for all $\mathrm{x} \in \mathrm{R}$. Clearly $\mathrm{R}$ is not comutative and $C=\{0\}$; moreover, $R$ has precisely four non-central subrings, $\langle a\rangle,\langle b\rangle$, $\langle C>$, and of course, $R$ itself.

THEOREM 5. If $R$ has no non-zero nilpotent elements and only finitely many non-central subrings, then $R$ is cormutative.

We shall make use of the following lemma. 
LEMMA 5. Let $R$ be a ring without non-zero divisors of zero. If $y \in R$ and there exist relatively prime integers $m, n$ such that $y^{m} \in C$ and $y^{n} \in C$, then $y \in C$.

PROOF. Let $y$ be a non-zero element of $R$ that satisfies our hypothesis. Assume without loss that $n>m$, and write $n=m q+r$ with $0<r<m$ and $q$ a positive integer. Observe that for all $x$ in $R$,

$$
0=\left[x, y^{n}\right]=\left[x, y^{m+r}\right]=\left[x, y^{m q}\right] y^{r}+y^{m q}\left[x, y^{r}\right] \text {. }
$$

Since $y^{m q} \in C$, it follows immediately that $y^{m q}\left[x, y^{r}\right]=0=\left[x, y^{r}\right]$. Therefore $y^{r} \in C$; and repeating the above argument for $m=q_{1} r+r_{1}$ and $0<r_{1}<r$, we get that $y^{r_{1}} \in$ c. Contimuing with the Euclidean algorithm until the remainder is 1 , we have that $y \in C$.

PROOF OF THECREM 5. We first note that $R$ is a subdirect product of rings with no non-zero divisors of zero [15], hence we may assume that $R$ is also without non-zero divisors of zero. Assume that there exists $y \in R \backslash C$. By Lemma 5, there exists an infinite sequence of integers $\mathrm{n}_{1}<\mathrm{n}_{2}<\mathrm{n}_{3}<\ldots$, denoted by $\Lambda$, such that $y^{\mathrm{n}_{i}} \mathrm{c}$ for all $\mathrm{n}_{\mathrm{i}}$ in $\Lambda$. Since the set $\left\{\left\langle y^{n_{i}}\right\rangle: n_{i} \in \Lambda\right\}$ contains only finitely many distinct subrings, there exist $n$ and $m$ in $\Lambda$, with $n\left\langle m\right.$, such that $\left\langle y^{n}\right\rangle=\left\langle y^{m}\right\rangle$. Hence $y^{n}=p\left(y^{m}\right)$, where $p(x) \in x z[x]$. Using the fact that $R$ has no non-zero divisors of zero, we see that $y=y^{2} q(y)$ for some $q(X) \epsilon z[X]$. Thus, for each $y \in R \backslash C$ there exists $q(X) \in z[X]$ such that $y-y^{2} q(y)$ is central; and since central elements obviously have the same property, $R$ is commutative by a well-known theorem of Herstein [16].

THEOREM 6. Let $R$ be a ring with $(R,+)$ torsion-free. If $R$ has only finitely many non-central subrings, then $R$ is commutative.

PROOF. Assuming that there exists a non-central element $y$, we again claim that there exists an infinite increasing sequence $\Lambda$ of positive integers such that $y^{n} \notin C$ for all $n \in \Lambda$. To see this, we need only suppose that our claim is false and hence there exists a positive integer $K$ such that for all integers $k \geq k, y^{k} \in C$. An argument similar to that used in proving Lemma 3 implies the existence of a non-zero integer $t$, and a polynomial $g(x) \in x^{k} z[X]$ such that $t^{K-1} y=g(y)$. Therefore $t^{K-1} y \in c$, which contradicts our assumption that $y \notin \mathrm{C}$.

consideration of $\{\langle y\rangle: n \in \Lambda\}$ again implies the existence of integers $\mathrm{n}$ and $\mathrm{m}$ in $\Lambda$, with $\mathrm{n}<\mathrm{m}$, and a polynomial $\mathrm{p}(\mathrm{X}) \in \mathrm{Z}[\mathrm{X}]$, such that $y^{n}=y^{m} p(y)$. Therefore $y^{n-1}\left(y-y^{m-n+1} p(y)\right)=0$, and a standard computation (cf. [5], proof of Lemma 3) shows that $y-y^{m-n+1} p(y)$ is in $N$. But Lemma 3 and the torsion-freeness of $(R,+)$ imply that $N \subseteq C$, and once again $R$ is commutative by Herstein's result. 
REMARK. Although a proof has eluded us thus far, we suspect that any ring with only a finite number of non-central subrings is finite or commutative.

5. RINGS WITH A FINITE MAXIMAL SUBRING.

T. J. Laffey has asked whether a ring with a finite maximal subring must be finite. The general question, which appears to be difficult, remains unanswered; but in interesting special cases, we have shown that the answer is affirmative.

THEOREM 7. Let $R$ have a finite maximal subring $M$ and at most finitely many subrings of infinite index. Then $R$ is finite.

PROOF. Let $I$ be any ideal not contained in $M$. Then $I+M$ is a subring properly containing $M$; and since $M$ is maximal, $I+M=R$. Thus we have the ring-theoretic isomorphism

$$
R / I=(I+M) / I \cong M /(M \cap I),
$$

and hence the index of $I$ is finite and bounded by $|M|$.

Note that $R$ is finitely-generated, since for any $x \notin M, R=\langle x, M\rangle$. A theorem of Lewin [17, Theorem 1], which asserts that a finitely-generated ring has only finitely many subrings of index a given integer, implies that $R$ can have only finitely many ideals not contained in $M$. Since $M$ is finite, we conclude that $R$ contains only finitely many ideals.

consider next a subring $S$ of finite index. Each such $S$ contains an ideal of finite index [17]; and since there are only finitely many such ideals, the index of $\mathrm{S}$ is bounded by the maximum of the set of indices of the ideals. Using Theorem 1 of [17] again, we have that R has only finitely many subrings of finite index. Hence $R$ has only finitely many subrings, and is therefore finite.

we conclude with

THEOREM 8. If $R$ is a commutative ring with a finite maximal subring $S$, then $R$ is finite.

PROOF. If $S$ is an ideal, then $R / S$ has no proper subrings; it is therefore finite, implying that $\mathrm{R}$ is also.

Let us assume that $R$ is a counterexample to the theorem with $|S|$ minimal among counterexamples. Consider the Jacobson radical $J(S)$, which is nilpotent, and $J(S) R+S$, which is a subring containing $S$. Maximality of $S$ implies that either $J(S) R+S=S$ or $J(S) R+S=R$. If we assume the latter, then

$$
R=(J(S) R+S) \subseteq(J(S)(J(S) R+S)+S) \subseteq J(S)^{2} R+S .
$$

Repetition of this argument shows that $R \subseteq J(S)^{n} R+S$ for all positive integers $n$, and the nilpotency of $J(S)$ then implies the ridiculous assertion that $R \subseteq S$. Therefore $J(S) R+S=S$, implying that $J(S) R$ is an ideal of $S$. Since $R$ is commutative, $J(S) R$ is nilpotent, which then implies that 
$J(S) R \subseteq J(S)$ and hence $J(S)$ is an ideal of $R$.

Note that $R / J(S)$ has a finite maximal subring $S / J(S)$. If $|S / J(S)|<|S|$, then, (by the minimality of $|S|$ ), R/J(S) is finite and $R$ is also. Therefore we must have that $J(S)=\{0\}$, so $S$ is non-nil and contains a non-zero idempotent $e$.

Assume temporarily that $e$ is a zero divisor in $R$, and suppose also that $\mathrm{eR} \notin \mathrm{S}$. Then $\mathrm{R}=\mathrm{eR}+\mathrm{S}$ and hence

$$
R / e R=(e R+S) / e R \cong S /(S \cap e R) \text {. }
$$

is finite. Since $R=e R \oplus A(e),(5.1)$ implies that $A(e)$ is finite. Considering es as a subring of $e R$, we observe that $e S=e R$ or es is a maximal subring of $e R$; and since we have assumed that $e R \nsubseteq S$, the latter must hold. If $|e s|<|S|$, then again by the minimality of $|S|$, eR is finite and $S O$ is $R$. Thus assume that $e S=S$, in which case the fact that $S=e S \oplus(A(e) \cap S)$ implies that $A(e) \cap S=\{0\}$. Because $A(e) \neq\{0\}$ and $S$ is a maximal subring, we have that $R=S+A(e)$, which implies that $R$ is finite.

Therefore we may assume that $e R \subseteq S$ - implying, of course, that $e R$ is finite. Since $e R$ is an ideal of $R$, our initial statement implies that $e R$ must be properly contained in $S$. Then $S / e R$ is a maximal subring of $R / e R$ with $|S / e R|<|S|$. Minimality of $|S|$ implies that $R / e R$ is finite and so is R.

It remains only to consider the case that all idempotents in $\mathrm{S}$ are regular in $R$. Since $J(S)=\{0\}, S$ is a direct product of finite fields; therefore, the regularity of idempotents forces $S$ to be a finite field. For any $x \in R \backslash S$, we have $\langle x, S\rangle=R=\{p(x): p(x) \in S[X]\}$; hence, if $x$ is algebraic over $S$, it is clear that $R$ is finite. On the other hand, if $x$ is not algebraic over $S$, then $R$ is isamorphic to $S[X]$, which does not contain a finite maximal subring. In any event, we have contradicted our assumption that $R$ was a counterexample.

ACKNOWLEDGEMENTS. H. E. Bell was supported by the Natural Sciences and Engineering Research Council of Canada, Grant No. A3961. F. Querriero was supported by an NSERC Undergraduate Student Research Award, Surmer 1988.

\section{REFERENCES}

1. PUTCHA, M. S. and YAQUB, A. Rings with a Finite Set of Nonnilpotents, Internat. J. Math. Math. Sci. 2 (1979), 121-126.

2. BELL, H. E. Same Conditions for Finiteness of a Ring, Internat. J. Math. Math. Sci. 11 (1988), 239-242.

3. BELL, H. E. A Cormutativity Study for Periodic Rings, Pacific J. Math. 70 (1977), 29-36. 
4. FUCHS, L. Abelian Groups, Pergamon Press, Oxford, 1960.

5. BELL, H. E. Infinite Subrings of Infinite Rings and Near-rings, Pacific J. Math. 59 (1975), 345-358.

6. LAFFEY, T. J. Infinite Rings with All Proper Subrings Finite, Amer. Math. Monthly 81 (1974), 270-274.

7. HERSTEIN, I. N. A Note on Rings with Central Nilpotent Elements, Proc. Amer. Math. Soc. 5 (1954), 620.

8. BELL, H. E. On Commutativity of Periodic Rings and Near-rings, Acta Math. Acad. Sci. Hungar. 36 (1980), 293-302.

9. SZELE, T. On a Finiteness Criterion for Modules, Publ. Math. Debrecen $\underline{3}$ (1954), 253-256.

10. LAFFEY, T. J. On Commutative Subrings of Infinite Rings, Bull. London Math. Soc. $\underline{4}$ (1972), 3-5.

11. OUTCALT, D. L. and YAQUB, A. A Comutativity Theorem for Rings, Bull. Austral. Math. Soc. 2 (1970), 95-99.

12. GANESAN, N. Properties of Rings with a Finite Number of zero Divisors, Math. Ann. 157 (1964), 215-218.

13. GANESAN, N. Properties of Rings with a Finite Number of Zero Divisors II, Math. Ann. 161 (1965), 241-246.

14. BELL, H. E. Rings with Finitely Many Subrings, Math. Ann. 182 (1969), 314-318.

15. BELL, H. E. Duo Rings: Same Applications to Commutativity Theorems, Canad. Math. Bull. 11 (1968), 375-380.

16. HERSTEIN, I. N. The Structure of a Certain Class of Rings, Amer. J. Math. 75 (1953), 864-871.

17. LEWIN, J. Subrings of Finite Index in Finitely-generated Rings, J. Algebra $\underline{5}$ (1967), 84-88. 


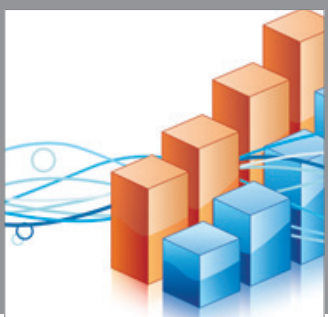

Advances in

Operations Research

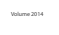

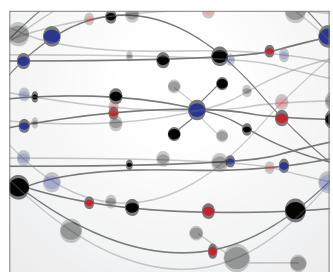

\section{The Scientific} World Journal
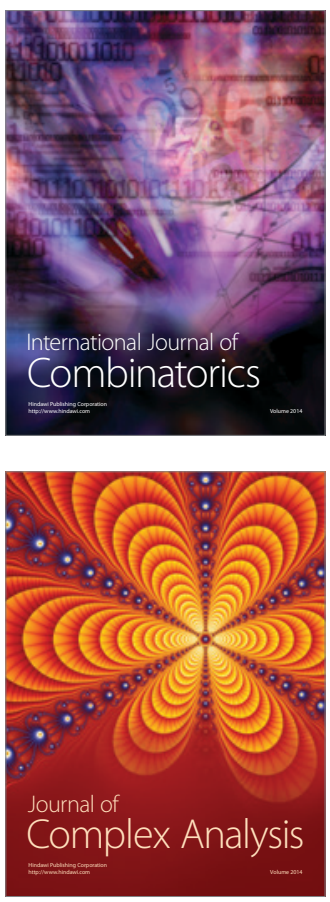

International Journal of

Mathematics and

Mathematical

Sciences
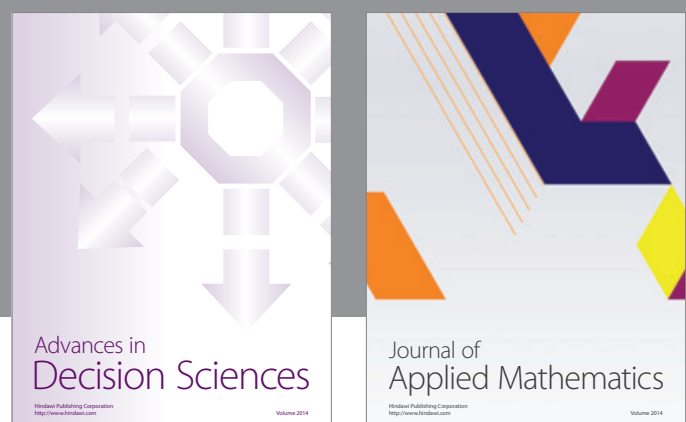

Journal of

Applied Mathematics
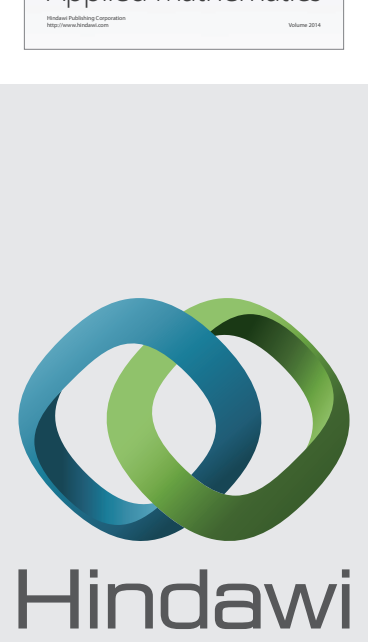

Submit your manuscripts at http://www.hindawi.com
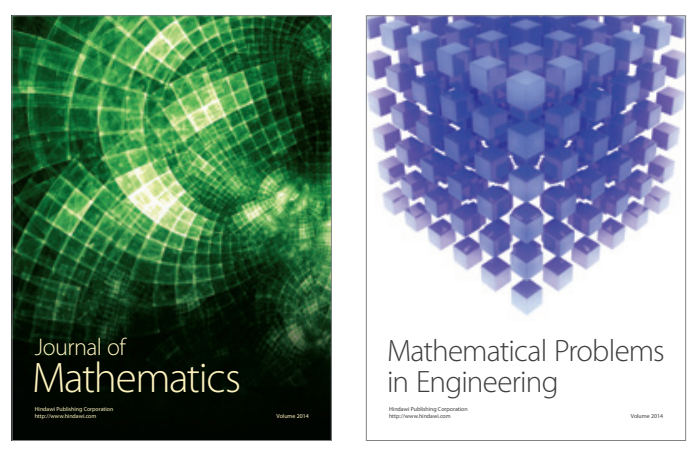

Mathematical Problems in Engineering
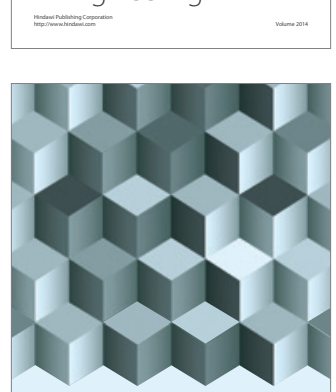

Journal of

Function Spaces
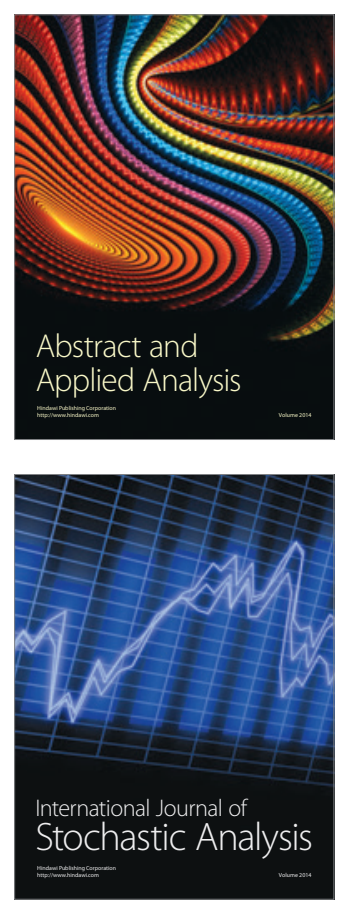

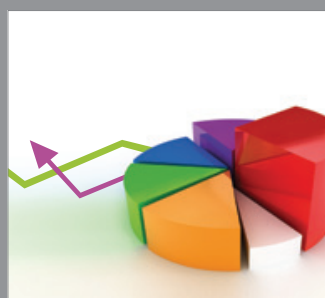

ournal of

Probability and Statistics

Promensencen
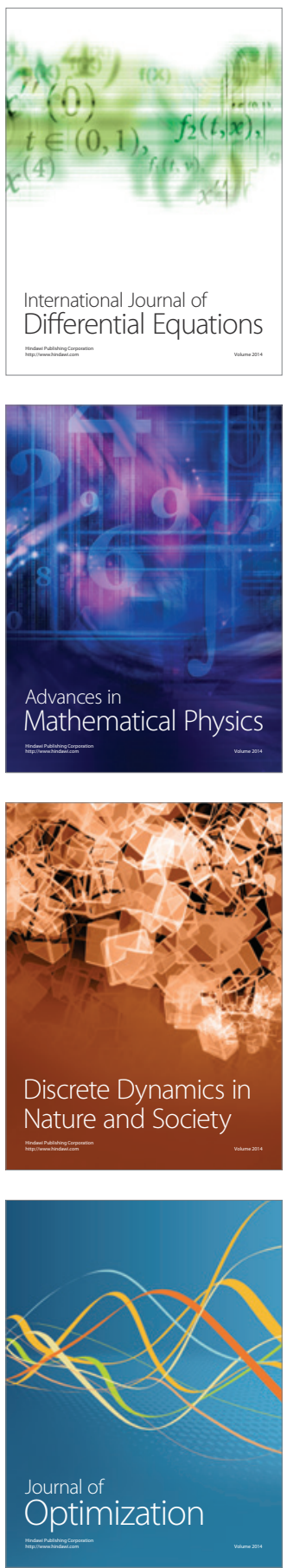